\title{
Chondroitin sulphation patterns in synovial fluid in osteoarthritis subsets
}

Samantha Lewis, Margot Crossman, Joanne Flannelly, Carolyn Belcher, Michael Doherty, Michael T Bayliss, Roger M Mason

\begin{abstract}
Objectives-To determine concentrations of chondroitin sulphate (CS) disaccharides in knee synovial fluid (SF) from normal subjects and patients with osteoarthritis (OA) or rheumatoid arthritis (RA), to test whether these variables differ between different diseases and subsets of OA.

Methods-OA was subdivided into large joint OA (LJOA), nodal generalised OA (NGOA), and OA with calcium pyrophosphate crystal deposition (CPA), with 25, 9, and 11 people in each subset respectively. The SF of 13 normal subjects was also volunteered for analysis along with 15 RA patients. Clinical assessment of inflammation (0-6) was undertaken on $O A$ and RA knees. Concentrations of unsaturated CS disaccharides $\Delta$ di6S and $\Delta$ di4S were measured by capillary zone electrophoresis.

Results-Concentrations of $\Delta$ di6S were lower in RA (5.90 $\mathrm{ng} / \mathrm{ml})$ and OA (13.24 $\mathrm{ng} / \mathrm{ml}$ ) fluids compared with normal (21.0 $\mathrm{ng} / \mathrm{ml}$ ) but no significant differences were seen between disease and normal fluids for $\Delta$ di4S (about 4-6 $\mathrm{ng} / \mathrm{ml}$ ). The ratio of $\Delta$ di6S: $\Delta$ di4S were $R A<O A<$ normal subjects ( $p<0.001$ for all comparisons). The disaccharide concentration values along with the ratios are below. Higher $\Delta$ di6S: $\Delta$ di4S ratios were obtained for $L J O A$ and CPA compared with NGOA. Uninflamed knees had lower concentrations of $\Delta$ di6S than inflamed knees $(p<0.01)$. In patients with bilateral samples, there were strong correlations between right and left knees for all SF variables.

Conclusions-Altered ratios of CS sulphation patterns occur in $O A$ and within $O A$ subsets. These further justify considering NGOA as a subset with a different aetiopathogenesis.

(Ann Rheum Dis 1999;58:441-445)
\end{abstract}

Glycosaminoglycans have been used to measure both anabolic and catabolic events in diseased joints. Thus, the expression of specific sulphation epitopes on chondroitin sulphate (CS) chains, recognised by monoclonal antibodies 3B3 and 7D4, have been linked to the biosyntheses of abnormal proteoglycans in pathological joint tissues. ${ }^{1}$ In contrast, measurements of total sulphated glycosaminoglycan and the keratan sulphate epitope $5 \mathrm{D} 4,{ }^{3}$ and more recent work with aggrecan CS epitope $846^{4}$ are generally accepted as indicators of connective tissue degradation. Changes in chondroitin sulphation were also exploited by Shinmei et $a \bar{l}$ in an attempt to differentiate between catabolic processes in inflammatory and noninflammatory arthritis. This was achieved by analysing the relative proportion of the unsaturated chondroitin sulphate disaccharides $\Delta$ di6S and $\Delta$ di $4 S$ released into synovial fluid after digesting CS with chondroitin lyase ABC. A more recent study extended this preliminary investigation and, by analysing the disaccharide content of normal synovial fluids as well as those from patients with joint disease, showed that the sulphation pattern was age, sex and disease related. ${ }^{6}$

In this study we have taken this approach a step further, to determine whether the concentration of unsaturated disaccharides in synovial fluid could also be used to differentiate between patients with different subsets of osteoarthritis (OA). OA is a heterogeneous condition and a number of subsets with possible differing pathogenesis have been suggested. We wish to determine whether there are biochemical differences between these subsets at the same effective site (the knee).

Department of

Sciences, The Royal

Veterinary College,

London

J Flannelly

M T Bayliss

Correspondence to:

Professor R M Mason.

Accepted for publication 26 March 1999

Table 1 Demographic details

\begin{tabular}{|c|c|c|c|c|c|}
\hline $\begin{array}{l}\text { Patient } \\
\text { group }\end{array}$ & $\begin{array}{l}\text { Number of } \\
\text { patients }\end{array}$ & Male:female & $\begin{array}{l}\text { Age (mean } \\
\text { (range)) }\end{array}$ & $\begin{array}{l}\text { Osteophyteformation } \\
\text { (median (IQ range)) }\end{array}$ & $\begin{array}{l}\text { Joint space narrowing } \\
\text { (median (IQ range)) }\end{array}$ \\
\hline$\overline{\mathrm{RA}}$ & 14 & $3: 11=1.0: 3.7$ & $57(28-85)$ & $1(1-3)(n=6)$ & $3(2-5)(n=6)$ \\
\hline Normal & 13 & $4: 9=1.0: 2.25$ & $34(21-75)$ & - & - \\
\hline LJOA & 25 & $13: 12=1.0: 1$ & $70(41-88)$ & $3.5(3-6)(n=14)$ & $3(2-4)(n=14)$ \\
\hline $\mathrm{CPA}$ & 11 & $8: 3=2.7: 1$ & $69(49-92)$ & $5(4-8)(\mathrm{n}=9)$ & $5(3-5)(n=9)$ \\
\hline NGOA & 9 & $2: 7=1.0: 3.5$ & $74(63-87)$ & $6(4-7)(n=9)$ & $4(3-5)(n=9)$ \\
\hline
\end{tabular}



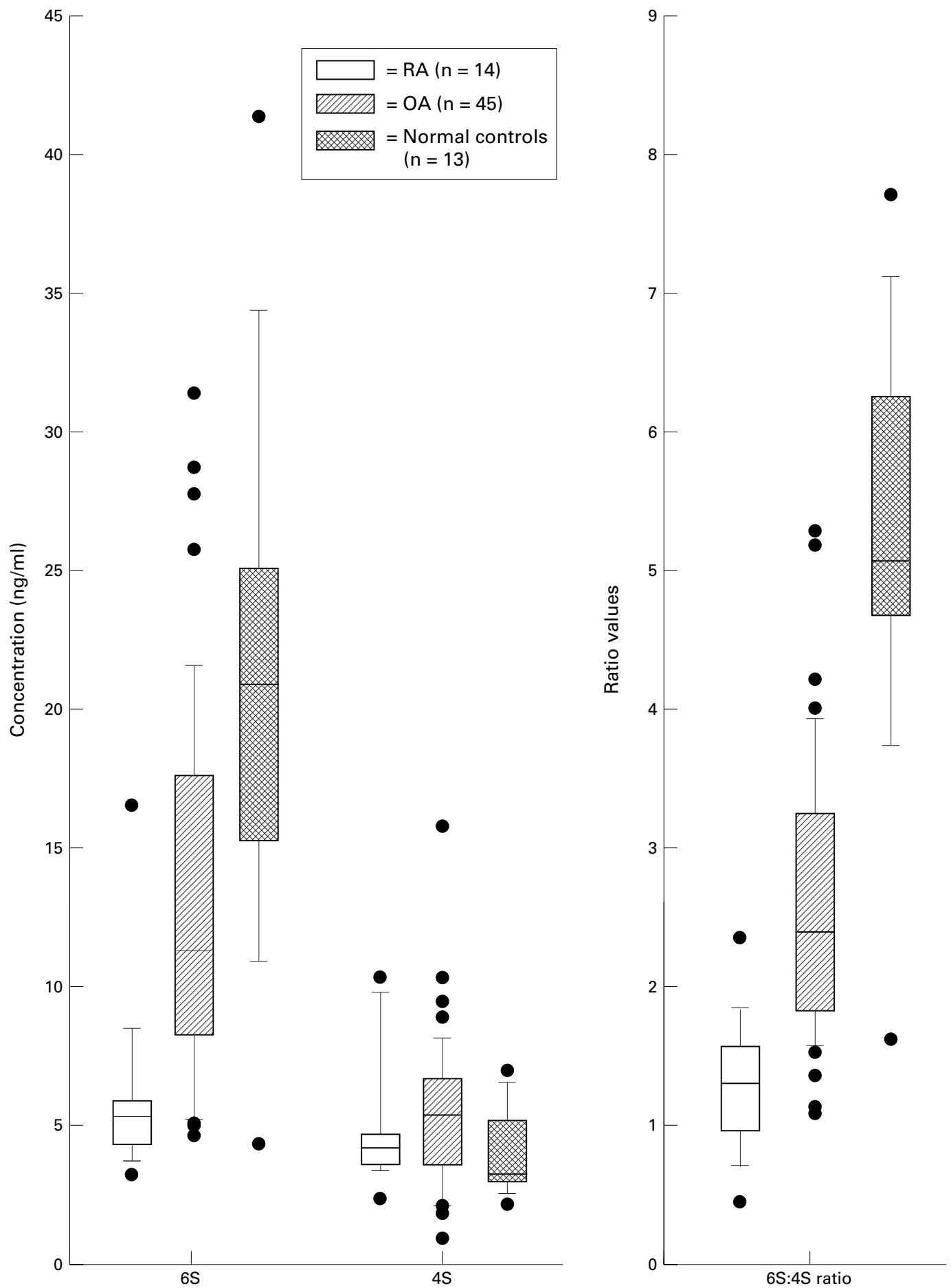

Figure 1 Comparison of the CS disaccharide composition of $S F$ from the $O A, R A$, and normal patient groups. $6 S=$ unsaturated 6-sulphated disaccharide; $4 S=$ unsaturated 4-sulphated disaccharide. The horizontal solid line represents the median, with the box representing the middle 50\% of the data. The error bar cap lines mark the 10th and 90th percentiles. Dots represent individual data points outside the 10th and 90th percentiles.

\section{Methods}

PATIENTS AND CLINICAL ASSESSMENT

Local research ethics committee approval was obtained for the study. Hospital referred patients with symptomatic knees affected by OA or rheumatoid arthritis (RA) were studied. Patients with knee OA all had radiographic evidence of joint space narrowing and osteophyte in one or more knee compartments (medial or lateral tibiofemoral, patellofemoral). Other joint pathology was excluded on the basis of full clinical assessment, radiographic features, synovial fluid (SF) examination and serological and biochemical tests. ${ }^{7}$
The RA patients all fulfilled ACR criteria for definite RA and all had symptoms and signs of knee involvement by RA. Radiological scoring was undertaken on subjects where SF was obtained within six months of an available radiograph, using the system previously described. ${ }^{7}$ For each of the three compartments, a score was given for narrowing (0-3) and osteophyte (0-3); summated scores for each knee were calculated for each feature (0-9). There was significant variation in the volume of SF aspirated from indivdual people, this variation was corrected for in the final calculations. 

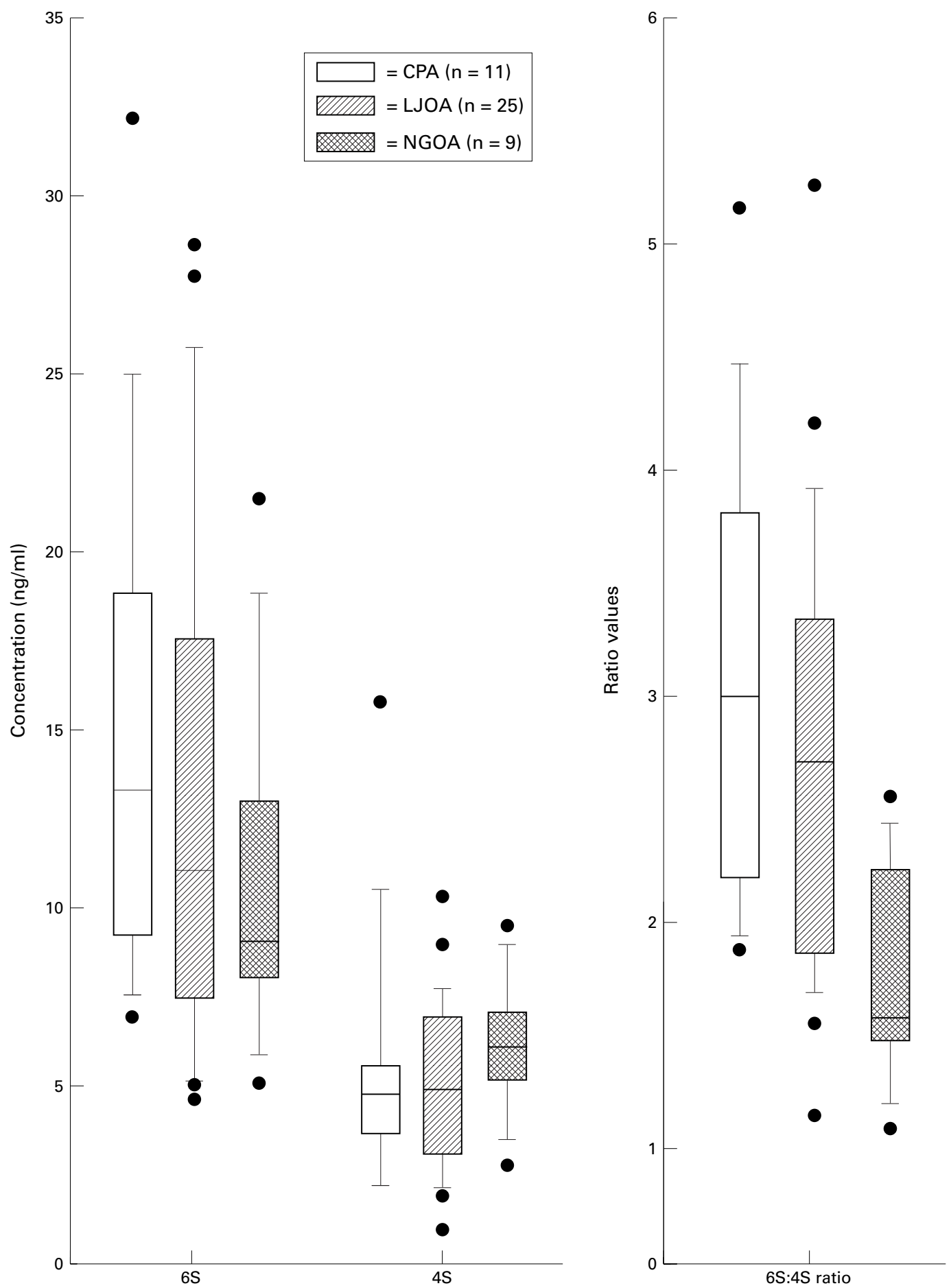

Figure 2 Comparison of the CS disaccharide composition of $S F$ from the $O A$ subgroups. The subgroups $L f O A=$ large joint $O A, N G O A=$ nodal generalised $O A$ and CPA = chronic pyrophosphate arthropathy. The horizontal solid line represents the median, with the box representing the middle $50 \%$ of the data. The error bar cap lines mark the 10th and 90 th percentiles. Dots represent individual data points outside the 10th and 90th percentiles.

The knee OA patients were subdivided into three subsets as previously. ${ }^{8}$

Pauciarticular large joint $O A$ (LFOA)

Knee OA with or without OA of other large joints (hips/shoulders), but no Heberden's nodes or clinical/radiographic evidence of polyarticular hand interphalangeal OA.

Nodal generalised $O A$ (NGOA)

Knee OA, plus Heberden's (with or without Bouchard's) nodes and radiographic evidence of interphalangeal OA (narrowing and/or osteophyte) affecting two or more rays of each hand.
Chronic pyrophosphate arthropathy (CPA)

Knee OA with calcium pyrophosphate dihydrate crystals identified in their knee SF (with or without radiographic chondrocalcinosis). Crystal identification was by compensated polarised light microscopy of fresh synovial fluid. Patients that overlapped these groups, for example those with NGOA and calcium pyrophosphate dihydrate crystals, were not included in the study.

For OA and RA knees clinical inflammation at the time of aspiration was designated "active" (score 4-6) or "inactive" (score 0-2) using a summated score of six clinical 
parameters: increased warmth, effusion, anterior joint line synovial thickening, joint line tenderness, early morning stiffness, inactivity stiffness. ${ }^{8}$ Knees scoring 3 were excluded. The components of this system show good interobserver and intraobserver reproducibility. ${ }^{9}$

SF was also obtained from knees of 13 healthy volunteers. Normal knees were defined as those that had never had symptoms (pain, stiffness, swelling) and that were normal on clinical examination, in a subject with no clinical evidence of OA or other arthropathy at other sites. Knee radiographs (standing anteroposterior and lateral 30 degrees flexion views) were obtained on subjects aged over 50 years to exclude occult pathology (for example, minor knee OA, chondrocalcinosis).

SYNOVIAL FLUIDS

Knees were aspirated to apparent dryness via a medial approach. A small sample of fresh SF was examined for crystals and the remainder collected into sterile plastic containers on ice, centrifuged at $2500 \mathrm{~g}$ for 15 minutes at $4^{\circ} \mathrm{C}$ and the supernatant stored at $-80^{\circ} \mathrm{C}$.

\section{UNSATURATED CS DISACCHARIDES $\triangle$ DI6S AND} $\triangle \mathrm{DI} 4 \mathrm{~S}$

Synovial fluids were sequentially digested with streptomyces hyaluronidase and chondroitin ABC lyase and the resultant unsaturated chondroitin disaccharides were analysed by capillary zone electrophoresis. ${ }^{10}$

STATISTICAL ANALYSIS

Comparison between disease groups was by Friedman two way analysis of variance and the Mann-Whitney U test with a Bonferroni correction. Correlations were calculated using Spearman's correlation coefficient.

\section{Results}

DEMOGRAPHIC DETAILS

Table 1 shows the subject numbers, ages and male:female ratios.Although the average age of the control group was younger than either of the OA or RA groups $(\mathrm{p}<0.05)$, the age range of all OA groups were similar. There were no significant differences between all OA groups for radiographic scores (osteophyte, joint space narrowing and radiograph total).

\section{DISACCHARIDE ANALYSIS}

\section{Correlation between left and right knees}

In patients where data were available on both knee joint SF, there were significant correlations between right and left joints for $\Delta$ di6S, $\Delta$ di4S or $\Delta$ di6S: $\Delta$ di4S ratio $(r=0.69-0.86$, $\mathrm{p}<0.001)$. Consequently, one knee was chosen at random for use in data analysis.

\section{Comparison of $R A, O A$, and normal groups}

There were distinct differences in the concentrations of the individual unsaturated disaccharides and in their ratios, between the control and patient groups and also within the patient groups. The concentration of $\Delta$ di6S was significantly lower in the RA SF than in the fluids from either OA patients $(p<0.001)$ or normal knee joints $(p<0.001)$ and it was also significantly lower in OA fluids compared with the normal controls $(\mathrm{p}<0.001)$ (fig 1). In marked contrast, there were no significant differences in the $\Delta$ di $4 \mathrm{~S}$ concentration of the control and disease groups. Thus, the ratios of $\Delta$ di6S: $\Delta$ di4S were significantly different when diseased fluids were compared with normal controls; $\mathrm{RA}<\mathrm{OA}<$ control $(\mathrm{p}<0.001$ for all comparisons).

Comparison of $O A$ subgroups

There were no differences between the OA subgroups in the concentration of $\Delta$ di6S or $\Delta$ di4S (fig 2). However, significantly higher $\Delta$ di6S: $\Delta$ di 4 S ratios were obtained for the LJOA and the CPA groups when compared with the NGOA group ( $\mathrm{p}<0.05$ in each case).

Comparison of $O A$ (active) and $O A$ (inactive) In OA knee joints scored as having active inflammation, there was a significantly lower concentration of $\Delta$ di6S $(p<0.05)$ than in inactive knee joints. The numbers of active and inactive in each OA subgroup were too small for statistical analysis.

Correlation of sulphation data with clinical scores for all $O A$ groups combined

There were no significant correlations between sulphation data and radiographic scores.

\section{Correlation of sulphation parameters with age in normal SF}

There was no significant correlation of any of the disaccharide values measured with the age of the person.

\section{Discussion}

Joint tissues, particularly articular cartilage, undergo various degrees of structural degeneration in OA and RA. There is also published evidence indicating that the metabolism of extracellular matrix components (collagen, proteoglycans and non-collagenous matrix proteins) is severely affected by the disease process and that products of this abnormal turnover are released into the SF. ${ }^{11}$ This physiological mechanism has provided the impetus for a number of studies investigating the mechanism(s) of tissue injury in joint disease. Using chemical and immunological methods to measure specific molecular fragments, temporal changes in various anabolic and catabolic pathways have been investigated. This approach has enabled novel hypotheses to be formulated concerning connective tissue repair and degeneration in arthritis. The objective of this study was not to identify a "marker" of a particular type of arthritic disease, but to investigate whether specific chondroitin sulphation patterns were associated with altered connective tissue metabolism when patients with classic OA of large joints had additional clinical and/or biochemical features associated with their joint disease.

The finding that the sum of chondroitin disaccharide $(\Delta$ di6S $+\Delta$ di4S $)$ concentrations in $\mathrm{OA}$ and RA joint fluids was significantly lower than those in normal fluids, suggests that the total mass of articular cartilage was decreased in 
the arthritic patients. These results are consistent with a study by Saxne $\mathrm{et} a \mathrm{l},{ }^{12}$ who found that there was an inverse correlation between cartilage destruction and the concentration of proteoglycan in SF. Similarly, the decreased ratio of $\Delta$ di6S: $\Delta$ di4S measured in the OA and RA fluids supported the analyses of Shinmei et $a \bar{P}$ and Sharif et al. ${ }^{6}$ However, the major new finding reported here is that the $\Delta$ di6S: $\Delta$ di $4 \mathrm{~S}$ ratio is significantly lower in patients who have nodal $\mathrm{OA}$ of the hand in addition to knee $\mathrm{OA}$, compared with those who only have large joint OA - that is, they have a synovial fluid composition that is more akin to that measured in RA SF. Although this suggests that similar biological mechanisms may be involved when nodal OA is present, it is worth noting that the presence of clinical inflammation in the OA groups did not affect the disaccharide values; the presence of pyrophosphate crystals, a known inflammatory mediator, in the SF did not affect the analyses. Furthermore, the significant correlation between the left and right knee joints for their SF analyses, as previously reported for SF hyaluronan, glycosaminoglycans, CS, keratan sulphate and pyrophosphate, ${ }^{813}$ suggest that these SF measures reflect an aspect of the patient rather than the local state of the joint. This is the second report of biochemical difference that has been detected in subsets of OA at the same anatomical site ${ }^{8}$ and is further justification for considering NGOA as a subset with a possibly different aetiopathogenesis.

Although the changes in SF composition have been discussed as if only articular cartilage were involved, the contribution of extra-articular tissues such as the meniscus, cruciate ligament and synovium to the SF pool should not be underestimated. ${ }^{14}$ These tissues also have CS containing proteoglycans in their extracellular matrix and their relative turnover in joint tissues is not known. However, in all arthritic joints soft tissues also show degenerative changes and the extent of these may contribute, in part, to the subgroup specific, SF composition.

This investigation demonstrates that disaccharide measurements of SF can provide useful information about the metabolic status of joint tissues and this analytical technique will be used in future studies to determine how temporal changes in joint disease and the effect of pharmaceutical intervention changes fluid composition. Funding: we would like to thank the Arthritis Research
Campaign, and The Oliver Bird Fund for Rheumatism for their generous financial support.

1 Caterson B, Griffin J, Mahmoodian F, Sorrell JM Monoclonal antibodies against chondroitin sulphate isomers: their use as probes for investigating proteoglycan metabolism. Biochem Soc Trans 1990;18:820-3.

2 Hazell PK, Dent C, Fairclough JA, Bayliss MT, Hardingham TE.Changes in glycosaminoglycan epitope levels in knee joint fluid following injury. Arthritis Rheum 1995; 38:953-9.

3 Thonar EJ, Masuda K, Hauselmann HJ, Uebelhart D, Lenz $\mathrm{ME}$, Manicourt DH. Keratan sulfate in body fluids in join disease. Acta Orthop Scand Suppl 1995;266:103-6.

4 Lundberg C, Asberg I, Ionescu M, Reiner A, Smedegard G Poole AR. Changes in cartilage proteoglycan aggrecan after intra-articular injection of interleukin-1 in rabbits: studies of synovial fluid and articular cartilage. Ann Rheum Dis 1996;55:525-34.

5 Shinmei M, Miyauchi S, Machida A, Miyazaki K. Quantitation of chondroitin 4-sulphate and chondroitin 6-sulphate in pathological joint fluid. Arthritis Rheum 1992;35: in patho

6 Sharif M, Osborne DJ, Meadows K, Woodhouse SM, Colvin EM, Shepstone L, et al. The relevance of chondroitin and keratan sulphate markers in normal and arthritic synovial fluid. Br J Rheumatol 1996;35:951-7.

7 Ledingham JM, Regan M, Jones A, Doherty M. Radiographic patterns and associations of knee osteoarthritis in a hospital referred population. Ann Rheum Dis 1993;52: 520-6.

8 Doherty M, Richards N, Hornby J, Powell R. Relationship between synovial fluid $\mathrm{C} 3$ degradation products and loca joint inflammation in rheumatoid arthritis, osteoarthritis and crystal associated arthropathy. Ann Rheum Dis 1988 47:190-7.

9 Jones A, Hopkinson N, Pattrick M, Berman P, Doherty M. Evaluation of a method for clinically assessing osteoarthritis of the knee. Ann Rheum Dis 1992;51:243-5.

10 Gaffen JD, Gleave S, Crossman MV, Bayliss MT, Mason RM. Articular cartilage proteoglycans in osteoarthritic STR/ort mice. Osteoarthritis Cartilage. 1995;3:95-104.

11 Heinegard D, Saxne T. Molecular markers of processes in cartilage in joint disease. Br J Rheumatol 1991;30:21-4.

12 Saxne T, Wollheim FA, Pettersson H, Heinegard D. Proteoglycan concentration in synovial fluid: predictor of future cartilage destruction in rheumatoid arthritis? BMJ 1987;295:1447-8 .

13 Doherty M, Belcher C, Regan M, Jones A, Ledingham J. Association between synovial fluid levels of inorganic pyrophosphate and short term radiographic outcome of osteoarthritis. Ann Rheum Dis 1996;55:432-6.

14 Bayliss MT, Davidson C, Woodhouse SM, Osborne DJ. Chondroitin sulphation in human joint tissues varies with age, zone and topography. Acta Orthop Scand 1995;66: $22-5$. 